\title{
Interventions to improve medicines optimisation in frail older patients in secondary and acute care settings: a systematic review of randomised controlled trials and non-randomised studies
}

\author{
Dima Saeed $^{1} \cdot$ Gillian Carter $^{2}$ D $\cdot$ Carole Parsons $^{1}$ (D)
}

Received: 9 July 2021 / Accepted: 9 November 2021 / Published online: 20 November 2021

(c) The Author(s) 2021

\begin{abstract}
Background: Frailty is a geriatric syndrome in which physiological systems have decreased reserve and resistance against stressors. Frailty is associated with polypharmacy, inappropriate prescribing and unfavourable clinical outcomes. Aim: To identify and evaluate randomised controlled trials (RCTs) and non-randomised studies of interventions designed to optimise the medications of frail older patients, aged 65 years and over, in secondary or acute care settings. Method: Literature searches were conducted across seven electronic databases and three trial registries from the date of inception to October 2021. All types of interventional studies were included. Study selection, data extraction, risk of bias and quality assessment were conducted by two independent reviewers. Results: Three RCTs were eligible for inclusion; two employed deprescribing as the intervention, and one used comprehensive geriatric assessment. All reported significant improvements in prescribing appropriateness. One study investigated the effect of the intervention on clinical outcomes including hospital presentations, falls, fracture, quality of life and mortality, and reported no significant differences in these outcomes, but did report a significant reduction in monthly medication cost. Two of the included studies were assessed as having 'some concerns' of bias, and one was judged to be at 'high risk' of bias. Conclusion: This systematic review demonstrates that medicines optimisation interventions may improve medication appropriateness in frail older inpatients. However, it highlights the paucity of high-quality evidence that examines the impact of medicines optimisation on quality of prescribing and clinical outcomes for frail older inpatients. High-quality studies are needed to address this gap.
\end{abstract}

Keywords Frailty $\cdot$ Frail elderly $\cdot$ Medicines optimisation $\cdot$ Medication review $\cdot$ Secondary care $\cdot$ Systematic review

\section{Impact on practice}

- Medicines optimisation interventions are safe, feasible and effective in improving the appropriateness of prescribing among frail hospitalised older patients.

- Hospitalised older patients should be screened for frailty using a validated instrument to inform the implementation of effective interventions in the context of the individual's morbidities, goals of care and life expectancy.

Carole Parsons

c.parsons@qub.ac.uk

1 School of Pharmacy, Queen's University Belfast, Belfast, UK

2 School of Nursing and Midwifery, Queen's University Belfast, Belfast, UK
- Medication review should be conducted regularly in frail older patients to evaluate whether medicines are providing ongoing net benefit or net harm.

\section{Background}

Frailty is a syndrome characterised by a cumulative decline across multiple physiological systems as well as decreased reserve and resistance against internal and external stressors; any small stress or minor illness may trigger a dramatic change in health status [1-3]. Frailty phenotype (FP) and Frailty index (FI) are the two major frailty models [3-5]. FP identifies frailty by the presence of three or more of five phenotypic criteria weakness, slowness, low level of physical activity, self-reported exhaustion, and unintentional weight loss [6]. FI defines frailty as cumulative age-related health deficits including physical, psychological, and social deficits 
[7]. About one-quarter of the population aged $\geq 50$ years across the world is frail [8]. Frailty is associated with unfavourable clinical outcomes including falls, hospitalisation, and mortality $[1,2,9,10]$.

Polypharmacy (the concurrent use of multiple medications) and potentially inappropriate prescribing (PIP) are highly prevalent among frail older patients who are at greater risk of experiencing more severe and frequent drugrelated negative outcomes than robust older adults [10-14]. PIP incorporates 'overprescribing' (the use of drugs without a valid indication), 'misprescribing' (the utilisation of incorrect drug, dose or route of administration) $[15,16]$ and 'underprescribing' (the omission of a potentially beneficial medication clinically indicated for the prevention of disease) [17-20], and refers to the prescribing of potentially inappropriate medications (PIMs), defined as medications with questionable efficacy that may cause significant risk of adverse drug reactions, excess morbidity and mortality $[15,21,22]$.

Acute admissions present valuable opportunities to review and optimise medicines for frail older people and to deprescribe PIMs in the context of an individual's morbidities, goals of care and life expectancy [11]. Medicines optimisation is "a person-centred approach to safe and effective medicines use, to ensure people obtain the best possible outcomes from their medicines" [23]. A regular medication review for frail patients allows evaluation of whether medications are providing ongoing net benefit or net harm $[11,24,25]$. Medication review is defined as a systematic and structural evaluation of a patient's pharmacotherapy to optimise medication use by identifying the medicationrelated problem and then changing the prescription [26, 27]. Targeted deprescribing among vulnerable populations is an important element of optimising medication. Deprescribing is defined as a process of addressing and stopping inappropriate medication. It encompasses medication withdrawal and reduction of medication dose and frequency [28-32]. A recent systematic review demonstrated that deprescribing among older people living with frailty in all types of healthcare settings significantly reduced the number of prescribed medications and PIMs [33]. However, little work has been conducted in hospitalised frail older patients to examine the impact of medicines optimisation interventions in secondary and acute care settings.

\section{Aim}

This systematic review aimed to identify and evaluate studies of interventions designed to optimise the medications of frail patients aged $\geq 65$ years in secondary or acute care settings and identify medication- and patient-related outcomes related to medicines optimisation reported in such studies.

\section{Method}

This systematic review was conducted in accordance with PRISMA (Preferred Reporting Items for Systematic Reviews and Meta-Analyses) 2020 guidelines [34] (Supplementary Material 1). The protocol was registered in the International Prospective Register of Systematic Reviews (PROSPERO) registry (registration number CRD42019156623) [35]. This review presents further analysis and discussion of preliminary findings presented previously [36].

\section{Search strategy}

Comprehensive literature searches were conducted to identify eligible studies and ongoing or completed clinical trials, published in English, from the date of inception to 13th October 2021. Seven electronic databases and three trial registries were searched: Medline, Scopus, Embase, Web of Science, International Pharmaceutical Abstracts, Cumulative Index to Nursing and Allied Health Literature Plus (CINAHL Plus), Cochrane Library, Cochrane Central Register of Controlled Trials, ClinicalTrials.gov, International Clinical Trials Registry Platform and Research Registry. According to the database being searched, search terms used were keywords, medical subject headings (MeSH) and EMTREE headings (Elsevier Life Science Thesaurus). Search terms were developed and refined with the assistance of a Queen's University Belfast subject librarian (search strategy is detailed in Supplementary Material 2). The reference lists of eligible studies were also hand-searched for any additional suitable articles that may have been missed during the database searches.

\section{Study selection and eligibility criteria}

All types of intervention studies aiming to optimise medications use in frail older inpatients (aged $\geq 65$ years) were eligible for inclusion in this review. The inclusion criteria for this review were guided by the population, intervention, comparator, outcome (PICO) framework [37], as outlined in Table 1.

Patients diagnosed with pre-frailty were not eligible for inclusion. Pre-frailty is an early and reversible risk-state before frailty [38] and is identified using the FP model by the presence of one or two of the five phenotypic criteria [6]. Additionally, multicomponent intervention studies where it was not possible to determine which component 
Table 1 Inclusion criteria for eligible studies

\begin{tabular}{ll}
\hline Population & - Frail older patients aged 65 years and over in secondary or acute care settings \\
- Frailty diagnosis using any existing frailty assessment tool \\
- Intervention relating to any aspect of 'Medicines Optimisation', 'Medicines Management', 'Pharmaceutical \\
Interventions \\
- Interventions delivered by any healthcare professional including geriatricians, pharmacists, nurses, or by a multi- \\
- disciplinary team \\
- Frail older inpatients (aged $\geq 65$ years) receiving: \\
a. Usual care (care as usually received by patients in everyday practice) or \\
b. No service (no intervention provided) \\
- Any change in medication (dose, frequency, dosage form, number of medications stopped or started) \\
- Appropriateness of prescribing \\
- Adverse drug reactions \\
- Death \\
- Quality of life \\
- Falls or recurrent falls \\
- Fractures \\
- Disability \\
- Cost of medication and/or cost of health care utilisation (i.e.hospital readmission and duration of hospitalisa- \\
tion) \\
- All types of randomised controlled trials (RCTs) \\
- Non-randomised studies (NRSs)
\end{tabular}

of the intervention was responsible for the reported outcomes were also excluded.

\section{Study selection}

After removing duplicates, initial assessment of the article titles and abstracts was conducted by the researcher (DS); studies that did not meet the inclusion criteria were excluded. Two reviewers (DS and $\mathrm{CP}$ ) independently reviewed the full text of all potentially eligible articles to determine if they met the inclusion criteria. Both reviewers discussed their results and a third reviewer (GC) was consulted if consensus could not be reached regarding including or excluding a study. A PRISMA 2020 flow diagram was generated to display the screening process and reasons for inclusion and exclusion of studies [34].

\section{Data extraction}

Data extraction was performed independently by two review authors (DS and CP) using the Cochrane data collection form as a template [39]. In the case of discrepancies, a third researcher (GC) was consulted. Study authors were contacted if data were missing.

\section{Quality assessment}

Two review authors (DS and CP) independently assessed the risk-of-bias of each study using the Cochrane Collaboration's Risk of Bias (ROB 2.0) tool for RCTs [40]. Disagreements were resolved by consensus and a third reviewer (GC) was consulted where necessary.

\section{Results}

\section{Study selection}

A total of 2041 articles were retrieved. After removal of duplicate publications, 1480 articles were screened for eligibility based on their titles and abstracts; full texts of 35 articles were assessed for eligibility. Of these, three RCTs were deemed eligible for inclusion [41-43]. Further studies were not identified from a manual search of the references of included studies. The study selection process and reasons for exclusion are summarised in Fig. 1, and the full list of excluded studies and reasons for exclusion are provided in Supplementary Material 3.

\section{Study characteristics}

The characteristics of the included studies are summarised in Table 2. Due to the heterogeneity of the included studies, meta-analysis was not possible, and a narrative synthesis was conducted. 
Fig. 1 Preferred Reporting Items for Systematic Reviews and Meta-Analyses (PRISMA 2020) flow diagram

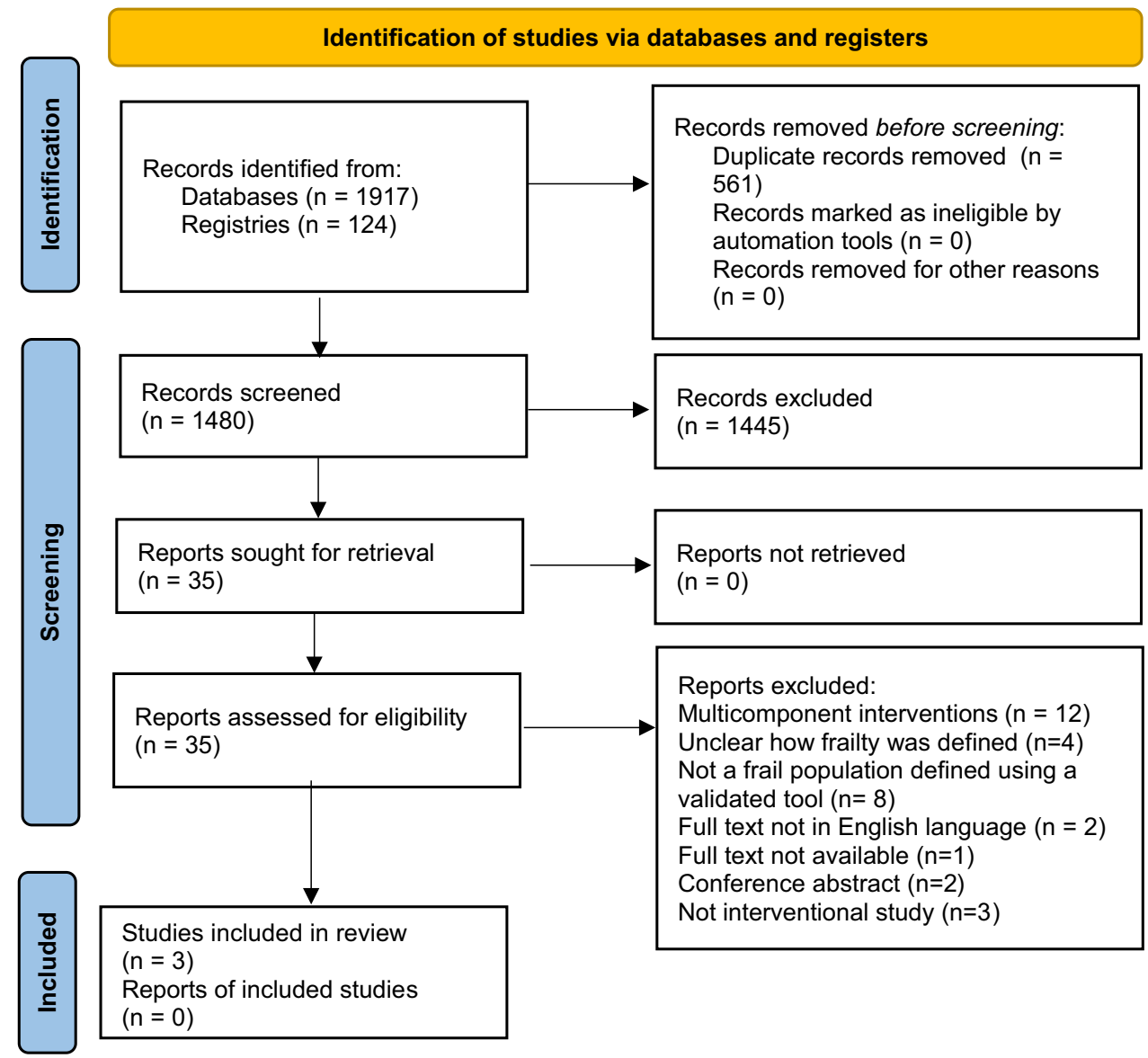

\section{Participants}

A total of 1133 hospitalised patients were recruited across the three included studies (mean sample size 378 participants, range 130-845). Curtin et al., explicitly targeted frail patients at the end of life and used the surprise question, whereby the clinician indicated that he or she "would not be surprised if the patient died in the next year"[44], to indicate one-year life expectancy [43].

\section{Characteristics of interventions}

The interventions in the included studies were delivered by a multidisciplinary team $(n=2)[41,42]$ or a physician $(n=1)$ [43]. The deprescribing interventions used a specific explicit tool in two studies; one [42] employed the Screening Tool of Older Person's Prescriptions (STOPP) criteria [20] and the other [43] employed the STOPPFrail criteria [45]. The third study employed geriatric evaluation and management according to published guidelines and Veterans Affairs (VA) hospital standards, whereby a comprehensive medication review was undertaken to assess all aspects of suboptimal prescribing (overuse, misuse, and underuse) and a combination of implicit and explicit PIM screening tools was utilised [41] including the Medication Appropriateness Index (MAI) [46], Beers Criteria [47] and the Assessment of Underutilization of Medication instrument [48].

\section{Effect of interventions: outcomes}

All included studies reported significant improvements in prescribing appropriateness [41-43]. Different outcomes were used as measures of prescribing appropriateness across the included studies, and included number of prescribed medications $(n=1)$ [43] and PIM discontinuation rate $(n=1)$ [42]. The third study reported several outcomes to cover all aspects of suboptimal prescribing, including the number of unnecessary medications, MAI score, number of PIMs according to the Beers criteria and number of conditions with omitted drugs [41] (Table 1). Two studies reported changes in prescribing of specific drug classes; one reported changes in prescription of antipsychotic medications and found that antipsychotic drugs were discontinued more often in intervention patients relative to control patients, however, 


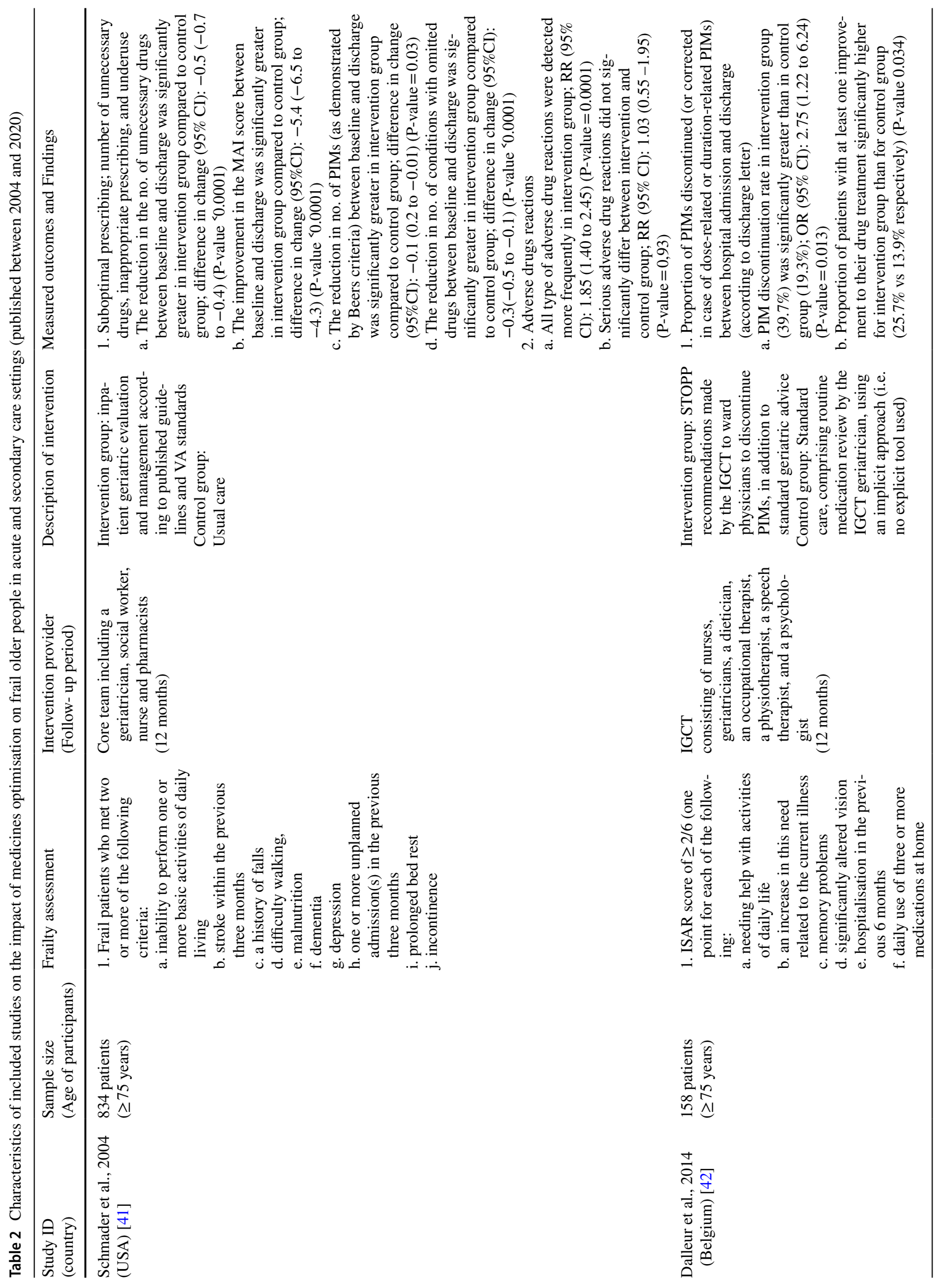




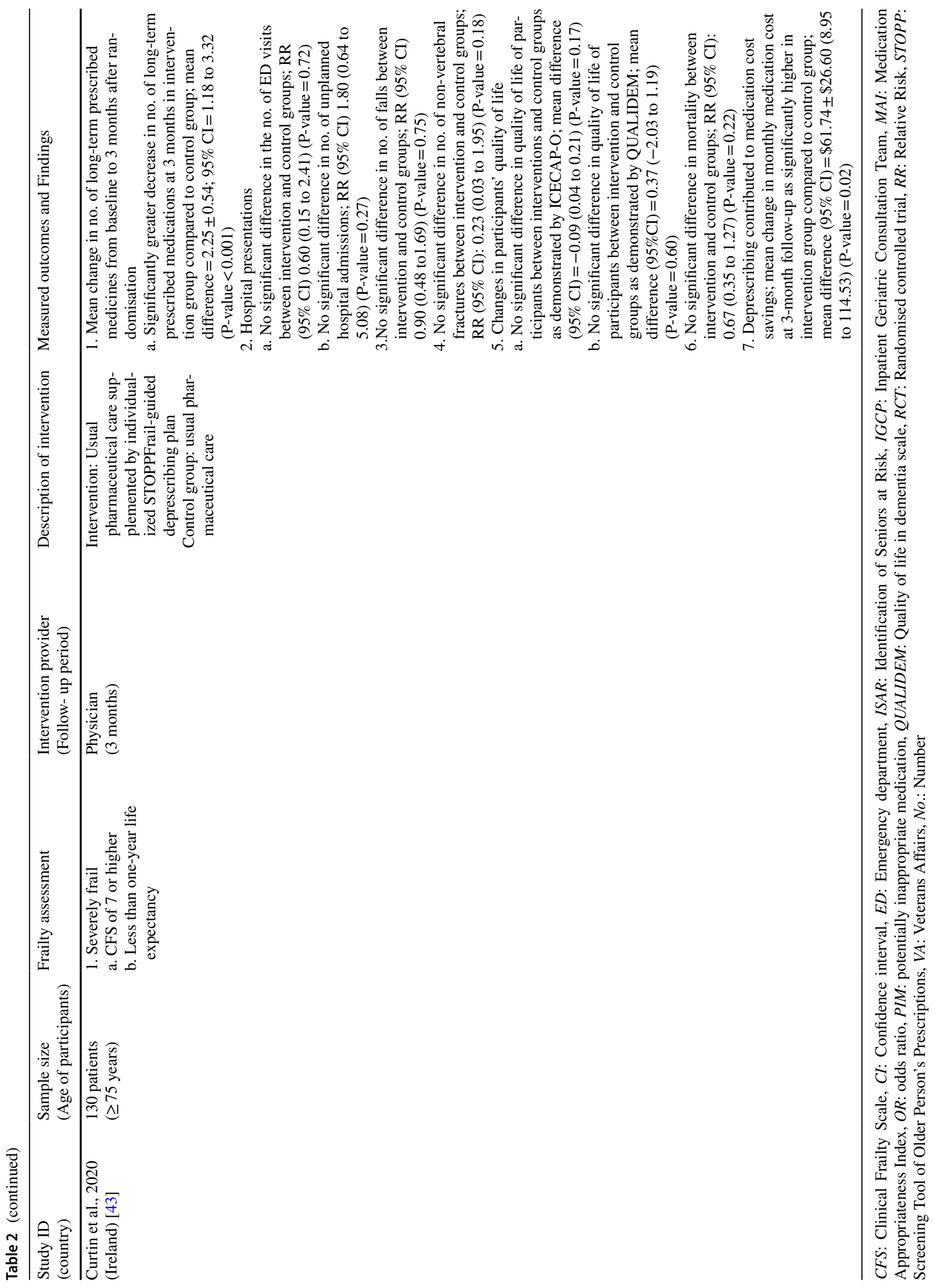


the difference did not reach statistical significance [43]. The second study reported PIM discontinuation rate for the most commonly deprescribed drug classes benzodiazepines, antiplatelets, opioids, $\beta$-blockers, tricyclic antidepressants and neuroleptics and found that the discontinuation rate was higher in the intervention group than in the control group. However, the $P$ value was only reported for benzodiazepines $(P$ value $=0.0063)[42]$.

Schmader et al. investigated the effect of medication review and optimisation on adverse drug reactions (ADRs) and reported that the number of all ADRs (minor plus serious) was significantly higher in the intervention group. However, there was no significant difference between intervention and control groups regarding the risk of serious ADRs.

Curtin et al. outlined the impact of deprescribing on health-related outcomes for frail older people after discharge, including quality of life (QoL), mortality, falls and fracture, unscheduled medical reviews and hospital presentations (emergency department visits not leading to admission, unplanned hospital admissions). No significant differences between intervention and control groups were observed for these outcomes (Table 2). However, this RCT was acknowledged by the authors as likely underpowered to detect differences in these outcomes [43].

The impact of deprescribing on medication cost was investigated by Curtin et al. [43] which reported that deprescribing contributed to medication cost savings; at 3-month follow-up, mean monthly medication cost savings were significantly greater for the intervention group than the control group.

\section{Quality assessment}

Risk of bias was assessed for prescribing appropriatenessrelated outcomes using ROB 2.0; results are summarised in Fig. 2. Two of the included studies were judged as having 'some concerns' of bias [41, 43], and one was judged as having a 'high-risk' of bias [42]. One study was judged as having 'some concerns' of bias associated with the randomisation process due to lack of reporting of allocation concealment [42]. None of the included studies reported sufficient details of analysis to estimate the effect of analysis (intention to treat analysis); all three studies were judged as having 'some concerns' in the 'deviations from intended interventions' domain. Curtin et al., (2020) was registered in ClinicalTrials.gov; the data were analysed according to a pre-specified plan and the study was judged to be at 'low risk of bias in the 'selection of the reported results' domain [43]. The other two studies were considered to have 'some concerns' in this domain [41, 42].

\section{Discussion}

\section{Statement of key findings}

This review highlights the paucity of evidence regarding the effectiveness of medicines optimisation interventions on outcomes for frail older inpatients in secondary and acute care settings; only three studies were included in this review. This is in line with findings of previous reviews which highlighted the lack of intervention studies aimed at improving appropriate polypharmacy in older people in general [49, 50]. Recruiting in acute settings is often challenging due
Fig. 2 Risk-of-bias summary: review authors' judgments about each risk of bias item for each included randomised controlled trial. (+) Low risk of bias; (-) High risk of bias; (?)

Some concerns of bias

\begin{tabular}{|c|c|c|c|c|c|c|}
\hline & 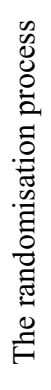 & 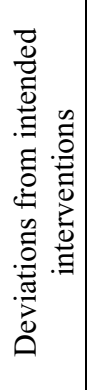 & 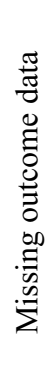 & 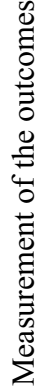 & 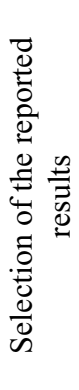 & $\begin{array}{l}n \\
0 \\
0 \\
\overline{0} \\
\bar{\pi} \\
0 \\
0\end{array}$ \\
\hline Schmader et al., 2004 & + & ? & + & + & & \\
\hline Dalleur et al., 2014 & & & & & & \\
\hline Curtin et al., 2020 & & & & & & \\
\hline
\end{tabular}

Low risk of bias Some concerns High risk of bias 
to acute illness, relatively short life expectancy, discharge before recruitment and data collection can be completed, difficulty in of follow-up post discharge, and lack of interest in participation [51].

Despite the recognition of the importance of frailty screening and assessment among older adults [52-55], many articles were excluded from this review at both abstract screening and full article screening stages because frailty was not diagnosed using a validated instrument. Several validated, simple, and quick assessment instruments are available and feasible to use in acute care [56-60] including the Clinical Frailty Scale [61], PRISMA 7 [62] and the Edmonton Frail Scale [63]. However, a lack of consensus definition for frailty and absence of agreement between frailty assessment instruments has hindered the wide implementation of these tools [52, 64].

Our systematic review assessed the impact of medicines optimisation interventions on prescribing in frail older inpatients and found that these interventions improved prescribing by reducing the number of PIMs. A previous Cochrane review to examine the impact of interventions to improve the appropriateness of prescribing in older people, albeit not specific for frail older people, in all types of healthcare settings reported improvement in the appropriateness of prescribing [50]. A recent systematic review and metaanalysis of RCTs to investigate the impact of different types of medicines optimisation interventions among older people living in residential care facilities or nursing homes found a significant improvement in medication appropriateness as demonstrated by reductions in the number of PIMs and the MAI score [65].

Two of the included studies reported that deprescribing reduced the number of regular medicines and PIMs consumed by frail older people [42, 43], and one reported that deprescribing did not adversely affect the rate of hospitalisation or mortality [43]. This reflects similar findings reported in a recent systematic review of deprescribing for frail older people in all types of healthcare settings, which demonstrated the feasibility, safety, tolerability and effectiveness of deprescribing in reducing the number of prescribed medications and number of PIMs [33]. These findings are also consistent with the findings of several other systematic reviews that examine the impact of deprescribing among older people in primary and secondary care settings as well as community-dwelling older adults [66-68].

Schmader et al. addressed the impact of medicines optimisation interventions on all aspects of PIP, including underprescribing [41], with the other two studies focusing on reducing the number of prescribed medications without addressing underuse [42, 43]. This is consistent with the findings of the Cochrane review of interventions to improve the appropriate use of polypharmacy in older people in any healthcare setting, which highlighted a dearth of evidence that assesses underprescribing [50] despite the prevalence of underprescribing among older populations $[17,19,20,69]$ and its association with negative health outcomes including increased morbidity, disability, healthcare utilisation, costs, and mortality [18].

Our review also assessed the impact of medicines optimisation interventions on clinical outcomes including falls, fractures, hospital presentation, QoL and mortality. The Curtin et al. study examined these outcomes and reported no significant differences in any measured outcome, but was not powered to evaluate any of these clinical outcomes [43]., Previous reviews in different clinical settings examined the impact of different types of medicines optimisation interventions in older patients and found limited evidence to drive strong conclusions about the impact on clinical outcomes; similarly, the majority of studies were not powered to detect significant differences [50,65, 66, 70-73]. This gap could be addressed through adequately powered studies designed to specifically evaluate any of these clinical outcomes. A recent systematic review of pharmacist-led interventions in older adults identified three RCTs out of 35 included studies which were adequately powered to detect differences in clinical outcomes. These RCTs found a significant association between a pharmacist-led intervention in older adults and a reduction in post-discharge hospital visits [74].

There is growing financial pressure on the healthcare system to meet the needs of older patients, particularly frail patients, to support them to maintain independent and healthier lifestyles [75, 76]. Consequently, cost-effectiveness data are required to increase the chances of successful implementation of any medicines optimisation service and to ensure effective resource utilisation. One study in this review examined the impact of deprescribing on medication costs and demonstrated a significant reduction [43]. This is in line with the findings of a previous systematic review and interventional studies conducted in different healthcare settings including nursing homes, intermediate and acute care settings, which found that medication review and deprescribing among older people generated substantial cost savings and a reduction in medication costs [33, 77-86]. However, the cost-effectiveness of medicines optimisation is still unclear and many reviews have highlighted the paucity of evidence regarding the economic impact of these interventions [87-89].

\section{Strengths and weaknesses}

This is the first systematic review to specifically investigate the impact of medicines optimisation interventions on prescribing and clinical outcomes for hospitalised frail older patients. Several systematic reviews have examined the impact of medicines optimisation interventions on PIMs 
and clinical outcomes in older patients in heterogeneous settings. However, the included studies did not explicitly target frail patients in whom frailty was assessed using a validated frailty tool $[33,50,65,66,70,90,91]$. A further strength of this systematic review was the rigorous methodology employed; a comprehensive search of large databases and trial registries was undertaken to include all types of studies and ongoing studies, with two reviewers independently screening all retrieved studies for inclusion. However, several limitations must be acknowledged when interpreting the findings of this systematic review. Explicitly including studies published in English may have led to language bias. Due to significant heterogeneity in intervention type and outcome measures, it was not possible to conduct a meta-analysis, and robust conclusions on efficacy by intervention type could not be made due to the limited number of included studies. Furthermore, the narrative synthesis presented should be treated with caution due to the small number and low quality of the included studies; none of the included studies were judged to be at low risk of bias and one was judged to be at 'high risk' of bias.

\section{Interpretation and further research}

Medicines optimisation is a safe, feasible, and effective approach to improve the appropriateness of prescribing in the acute care settings. High-quality studies are needed to outline the cost-effectiveness and impact of medicines optimisation for frail hospitalised older patients. Strategies that facilitate the identification of frail patients in hospitals and the recruitment of these patients in clinical trials should also be implemented.

\section{Conclusion}

This systematic review highlights the paucity and the low quality of evidence examining the impact of medicines optimisation on quality of prescribing and clinical outcomes for frail older inpatients which limits our ability to draw robust conclusions. It suggests that medicines optimisation interventions may improve prescribing appropriateness in frail older inpatients. However, although their impact on frail patients' clinical outcomes is unclear, these interventions seem to be safe and feasible for implementation in acute settings.

Supplementary Information The online version contains supplementary material available at https://doi.org/10.1007/s11096-021-01354-8.
Acknowledgements The authors would like to thank the Queen's University Belfast subject librarian, Angela Thompson, for her invaluable contribution in developing the search strategy.

Funding The authors received no specific funding for this work.

Data availability Data derived from sources in the public domain. All data were obtained from published papers.

Conflict of interest The authors declare that they have no conflicts of interest that are relevant to the content of this article.

Open Access This article is licensed under a Creative Commons Attribution 4.0 International License, which permits use, sharing, adaptation, distribution and reproduction in any medium or format, as long as you give appropriate credit to the original author(s) and the source, provide a link to the Creative Commons licence, and indicate if changes were made. The images or other third party material in this article are included in the article's Creative Commons licence, unless indicated otherwise in a credit line to the material. If material is not included in the article's Creative Commons licence and your intended use is not permitted by statutory regulation or exceeds the permitted use, you will need to obtain permission directly from the copyright holder. To view a copy of this licence, visit http://creativecommons.org/licenses/by/4.0/.

\section{References}

1. Fulop T, Larbi A, Witkowski JM, et al. Aging, frailty and agerelated diseases. Biogerontology. 2010;11(5):547-63.

2. Fried LP, Tangen CM, Walston J, et al. Frailty in older adults: evidence for a phenotype. J Gerontol A Biol Sci Med Sci. 2011;56(3):M146-57.

3. Clegg A, Young J, Iliffe S, Rikket MO, Rockwood K. Frailty in older people. Lancet. 2013;381(9868):752-62.

4. Chen X, Mao G, Leng SX. Frailty syndrome: an overview. Clin Interv Aging. 2014;9:433-41.

5. Walston JD, Bandeen-Roche K. Frailty: a tale of two concepts. BMC Med. 2015;13(1):6-8.

6. Fried LP, Tangen CM, Walston J, et al. Frailty in older adults: evidence for a phenotype. J Gerontol Ser A Biol Sci Med Sci. 2001;56(3):146-57.

7. Rockwood K, Mitnitski A. Frailty in relation to the accumulation of deficits. J Gerontol Ser A Biol Sci Med Sci. 2007;62(7):722-7.

8. O'Caoimh R, Sezgin D, O'Donovan MR, et al. Prevalence of frailty in 62 countries across the world: a systematic review and meta-analysis of population-level studies. Age Ageing. 2021;50(1):96-104.

9. Gobbens RJJ, van Assen MALM, Luijkx KG, et al. Determinants of frailty. J Am Med Dir Assoc. 2010;11(5):356-64.

10. Bennett A, Gnjidic D, Gillett M, et al. Prevalence and impact of fall-risk-increasing drugs, polypharmacy, and drug-drug interactions in robust versus frail hospitalised falls patients: a prospective cohort study. Drugs Aging. 2014;31(3):225-32.

11. Hilmer SN, Gnjidic D. Prescribing for frail older people. Aust Prescr. 2017;40(5):174-8.

12. Moulis F, Moulis G, Balardy L, et al. Searching for a polypharmacy threshold associated with frailty. J Am Med Dir Assoc. 2015;16(3):259-61.

13. Herr M, Sirven N, Grondin H, et al. Frailty, polypharmacy, and potentially inappropriate medications in old people: findings in a representative sample of the French population. Eur J Clin Pharmacol. 2017;73(9):1165-72. 
14. Veronese N, Stubbs B, Noale M, et al. Polypharmacy is associated with higher frailty risk in older people: an 8-Year longitudinal cohort study. J Am Med Dir Assoc. 2017;18(7):624-8.

15. Spinewine A, Schmader KE, Barber N, et al. Appropriate prescribing in elderly people: how well can it be measured and optimised? Lancet. 2007. https://doi.org/10.1016/S01406736(07)61091-5.

16. Curtin D, Gallagher PF, Denis O, et al. Explicit criteria as clinical tools to minimize inappropriate medication use and its consequences. Ther Adv Drug Saf. 2019;10(6):259-61.

17. O'mahony D, O'sullivan D, Byrne S, et al. STOPP/START criteria for potentially inappropriate prescribing in older people: Version 2. Age Ageing. 2015;44(2):213-8.

18. Cherubini A, Corsonello A, Lattanzio F. Underprescription of beneficial medicines in older people: causes, consequences and prevention. Drugs Aging. 2012;29(6):463-75.

19. Lombardi F, Paoletti L, Carrieri B, et al. Underprescription of medications in older adults: causes, consequences and solutions-a narrative review. Eur Geriatr Med. 2021. https://doi.org/ 10.1007/s41999-021-00471-x.

20. Gallagher P, Ryan C, Byrne S, et al. STOPP (Screening Tool of Older Person's Prescriptions) and START (Screening Tool to Alert doctors to Right Treatment) Consensus validation. Int J Clin Pharmacol Ther. 2008;46(2):72-83.

21. O'Connor MN, Gallagher P, O'Mahony D. Inappropriate prescribing: criteria, detection and prevention. Drugs Aging. 2012;29(6):437-52.

22. Tosato M, Landi F, Martone AM, et al. Potentially inappropriate drug use among hospitalised older adults: results from the CRIME study. Age Ageing. 2014;43(6):767-73.

23. National Institute for Health and Care Excellence (NICE). Medicines optimisation: the safe and effective use of medicines to enable the best possible outcomes.2015. https://www.nice.org. uk/guidance/ng5/resources/medicines-optimisation-the-safe-andeffective-use-of-medicines-to-enable-the-best-possible-outcomespdf-51041805253. Accessed on 24.04.2021

24. Hilmer SN, Gnjidic D, Le Couteur DG. Thinking through the medication list-appropriate prescribing and deprescribing in robust and frail older patients. Aust Fam Phys. 2012;41(12):924-8.

25. Récoché I, Lebaudy C, $\mathrm{Cool} \mathrm{C}$, et al. Potentially inappropriate prescribing in a population of frail elderly people. Int J Clin Pharm. 2017;39(1):113-9.

26. Christensen M, Lundh A. Medication review in hospitalised patients to reduce morbidity and mortality. CDSR. 2016. https:// doi.org/10.1002/14651858.CD008986.pub3.

27. Griese-Mammen N, Hersberger KE, Messerli M, et al. PCNE definition of medication review: reaching agreement. Int J Clin Pharm. 2018;40(5):1199-208.

28. Scott IA, Hilmer SN, Reeve E, et al. Reducing inappropriate polypharmacy: the process of deprescribing. JAMA Intern Med. 2015;175(5):827-34.

29. McGrath K, Hajjar ER, Kumar C, et al. Deprescribing: a simple method for reducing polypharmacy. J Fam Pract. 2017;66(7):436-45.

30. Bemben NM. Deprescribing: an application to medication management in older adults. Pharmacotherapy. 2016;36(7):774-80.

31. Dowden A. Deprescribing: reducing inappropriate polypharmacy. Prescriber. 2017;28(2):45-9.

32. Reeve E, Gnjidic D, Long J, et al. A systematic review of the emerging definition of "deprescribing" with network analysis: implications for future research and clinical practice. Br J Clin Pharmacol. 2015;80(6):1254-68.

33. Ibrahim K, Cox NJ, Stevenson JM, et al. A systematic review of the evidence for deprescribing interventions among older people living with frailty. BMC Geriatr. 2021;21(1):258.
34. Page MJ, McKenzie JE, Bossuyt PM, et al. The PRISMA 2020 statement: an updated guideline for reporting systematic reviews. BMJ. 2021;10(1):1-15.

35. Saeed D, Gillian C, Parsons C. Interventions to improve medicines optimisation in frail older patients in secondary and acute care settings: protocol. PROSPERO 2019 CRD42019156623. https:// www.crd.york.ac.uk/prospero/display_record.php?ID=CRD42 019156623. Accessed 25 January 2021.

36. Saeed D, Carter G, Parsons C. A systematic review of interventions to improve medicines optimisation in frail older patients in secondary and acute care settings. Int J Pharm Pract. 2021;29(SUPPL. 1):i22-3.

37. Thomas J, Kneale D, McKenzie JE, et al. Chapter 2: determining the scope of the review and the questions it will address. In: Higgins JPT, Thomas J, Chandler J, Cumpston M, Li T, Page MJ, Welch VA (editors). Cochrane Handbook for Systematic Reviews of Interventions version 6.2 (updated February 2021). Cochrane, 2021. Available from www.training.cochrane.org/handbook. Accessed 22.04.2021

38. Sezgin D, Liew A, O'Donovan MR, et al. Pre-frailty as a multidimensional construct: a systematic review of definitions in the scientific literature. Geriatr Nurs (Minneap). 2020;41(2):139-46.

39. Cochrane Developmental, Psychosocial and learning problems data collection form for intervention reviews: RCTs and nonRCTs. Cochrane 2021. Available from https://dplp.cochrane.org/ data-extraction-forms. Accessed 22.04.2021

40. Sterne JAC, Savović J, Page MJ, et al. RoB 2: a revised tool for assessing risk of bias in randomised trials. BMJ. 2019;366:1-8.

41. Schmader KE, Hanlon JT, Pieper CF, et al. Effects of geriatric evaluation and management on adverse drug reactions and suboptimal prescribing in the frail elderly. Am J Med. 2004;116(6):394-401.

42. Dalleur O, Boland B, Losseau C, et al. Reduction of potentially inappropriate medications using the STOPP criteria in frail older inpatients: a randomised controlled study. Drugs Aging. 2014;31(4):291-8.

43. Curtin D, Jennings E, Daunt R, et al. Deprescribing in older people approaching end of life: a randomized controlled trial using STOPPFrail Criteria. J Am Geriatr Soc. 2020;68(4):762-9.

44. White N, Kupeli N, Vickerstaff V, et al. How accurate is the "Surprise Question" at identifying patients at the end of life? A systematic review and meta-analysis. BMC Med. 2017;15(1)

45. Lavan AH, Gallagher P, Parsons C, O’Mahony D. STOPPFrail (Screening tool of older persons prescriptions in frail adults with limited life expectancy): consensus validation. Age Ageing. 2017;46(4):600-7.

46. Hanlon JT, Schmader KE, Samsa GP, et al. A method for assessing drug therapy appropriateness. J Clin Epidemiol. 1992;45(10):1045-51.

47. Beers MH. Explicit criteria for determining potentially inappropriate medication use by the elderly. An update. Arch Intern Med. 1997;157(14):1531-6.

48. Jeffery S, Ruby CM, Twesky J, et al. Effect of an interdisciplinary team on suboptimal prescribing in a long-term care facility. Consult Pharm. 1999; http://www.epistemonikos.org/documents/ 32bc2cf9b5cfec703b6f4e04497f5146cf4581ce

49. Ryan R, Santesso N, Lowe D, et al. Interventions to improve safe and effective medicines use by consumers: an overview of systematic reviews. CDSR. 2014. https://doi.org/10.1002/14651858. CD007768.pub3.

50. Rankin A, Cadogan CA, Patterson SM, et al. Interventions to improve the appropriate use of polypharmacy for older people. CDSR. 2018. https://doi.org/10.1002/14651858.CD008165.pub4.

51. McMurdo ME, Roberts $\mathrm{H}$, Parker $\mathrm{S}$, et al. Improving recruitment of older people to research through good practice. Age Ageing. 2011;40(6):659-65. 
52. van Abellan Kan G, Rolland Y, Bergman H, et al. The I.A.N.A Task Force on frailty assessment of older people in clinical practice. J Nutr Health Aging. 2008;12(1):29-37.

53. Morley JE, Vellas B, van Kan GA, et al. Frailty consensus: a call to action. J Am Med Dir Assoc. 2013;14(6):392-7.

54. Turner G, Clegg A. Best practice guidelines for the management of frailty: a British Geriatrics Society, Age UK and Royal College of General Practitioners report. Age Ageing. 2014;43(6):744-7.

55. Woolford SJ, Aggarwal P, Sheikh CJ, et al. Frailty, multimorbidity and polypharmacy. Medicines. 2021;49(3):166-72.

56. Hoffmann S, Wiben A, Kruse M, et al. Predictive validity of PRISMA-7 as a screening instrument for frailty in a hospital setting. BMJ Open. 2020. https://doi.org/10.1136/bmjop en-2020-038768.

57. Perna S, Francis MDA, Bologna C, et al. Performance of Edmonton Frail Scale on frailty assessment: its association with multi-dimensional geriatric conditions assessed with specific screening tools. BMC Geriatr. 2017. https://doi.org/10.1186/ s12877-016-0382-3.

58. Basic D, Shanley C. Frailty in an older inpatient population: using the clinical frailty scale to predict patient outcomes. J Aging Health. 2015;27(4):670-85.

59. Juma S, Taabazuing MM, Montero-Odasso M. Clinical frailty scale in an acute medicine unit: a simple tool that predicts length of stay. Can Geriatr J. 2016;19(2):34-9.

60. Church S, Rogers E, Rockwood K, et al. A scoping review of the clinical frailty scale. BMC Geriatr. 2020;20(1):393. https://doi. org/10.1186/s12877-020-01801-7.

61. Rockwood K, Song X, MacKnight C, et al. A global clinical measure of fitness and frailty in elderly people. CMAJ. 2005;173(5):489-95.

62. Hébert R, Durand PJ, Dubuc N, et al. PRISMA: a new model of integrated service delivery for the frail older people in Canada. Int J Integr Care. 2003;3(1):1-8.

63. Rolfson DB, Majumdar SR, Tsuyuki RT, et al. Validity and reliability of the Edmonton Frail Scale. Age Ageing. 2006;35(5):526-9.

64. Walston J, Buta B, Xue QL. Frailty Screening and Interventions: considerations for Clinical Practice. Clin Geriatr Med. 2018;34(1):25-38

65. Almutairi H, Stafford A, Etherton-Beer C, et al. Optimisation of medications used in residential aged care facilities: a systematic review and meta-analysis of randomised controlled trials. BMC Geriatr. 2020;20(1):1-19.

66. Thillainadesan J, Gnjidic D, Green S, et al. Impact of deprescribing interventions in older hospitalised patients on prescribing and clinical outcomes: a systematic review of randomised trials. Drugs Aging. 2018;35(4):303-19.

67. Thio SL, Nam J, Van Driel ML, et al. Effects of discontinuation of chronic medication in primary care: a systematic review of deprescribing trials. Br J Gen Pract. 2018;68(675):e663-72.

68. Ulley J, Harrop D, Ali A, et al. Deprescribing interventions and their impact on medication adherence in community-dwelling older adults with polypharmacy: a systematic review. BMC Geriatr. 2019;19(1):1-13.

69. San-José A, Agustí A, Vidal X, et al. Inappropriate prescribing to the oldest old patients admitted to hospital: prevalence, most frequently used medicines, and associated factors. BMC Geriatr. 2015;15(1):1-9.

70. Alldred DP, Kennedy MC, Hughes C, et al. Interventions to optimise prescribing for older people in care homes. CDSR. 2016;2(2):37-43.

71. Renaudin P, Boyer L, Esteve MA, et al. Do pharmacist-led medication reviews in hospitals help reduce hospital readmissions? A systematic review and meta-analysis. Br J Clin Pharmacol. 2016;82(6):1660-73.
72. Aprahamian I, Biella MM, Aricó Vano, de Almeida G, et al. Polypharmacy but not potential inappropriate prescription was associated with frailty in older adults from a middle-income country outpatient clinic. J Frailty Aging. 2018;7(2):108-12.

73. Tecklenborg $\mathrm{S}$, Byrne $\mathrm{C}$, Cahir $\mathrm{C}$, et al. Interventions to reduce adverse drug event-related outcomes in older adults: a systematic review and meta-analysis. Drugs Aging. 2020;37(2):91-8.

74. Van der Linden L, Hias J, Walgraeve K, et al. Clinical pharmacy services in older inpatients: an evidence-based review. Drugs Aging. 2020;37(3):161-74.

75. Iacobucci G. A service under pressure. BMJ. 2017;356:i6691.

76. Robertson R, Wenzel L, Thompson J, et al. Understanding NHS financial pressures: how are they affecting patient care? King's Fund. 2017. Available from https://www.kingsfund.org.uk/sites/ default/files/field/field_publication_summary/Understanding\% 20NHS\%20financial\%20pressures\%20-\%20report\%20summary. pdf. Accessed 25.04.2021

77. Roberts MS, Stokes JA, King MA, et al. Outcomes of a randomized controlled trial of a clinical pharmacy intervention in 52 nursing homes. Br J Clin Pharmacol. 2001;51(3):257-65.

78. Saeed D, Miller R, Darcy C, et al. Service evaluation of the medicines optimisation in older people (MOOP) service for frail older patients in the acute care setting. Int J Pharm Pract. 2020 (Suppl_1) 4-43

79. Pope G, Wall N, Peters CM, et al. Specialist medication review does not benefit short-term outcomes and net costs in continuingcare patients. Age Ageing. 2011;40(3):307-12.

80. Furniss L, Burns A, Craig SKL, et al. Effects of a pharmacist's medication review in nursing homes Randomised controlled trial. Br J Psychiatry. 2000;176:563-7.

81. Crotty M, Halbert J, Rowett D, et al. An outreach geriatric medication advisory service in residential aged care: a randomised controlled trial of case conferencing. Age Ageing. 2004;33(6):612-7.

82. Frankenthal D, Lerman Y, Kalendaryev E, et al. Intervention with the screening tool of older persons potentially inappropriate prescriptions/screening tool to alert doctors to right treatment criteria in elderly residents of a chronic geriatric facility: a randomized clinical trial. J Am Geriatr Soc. 2014;62(9):1658-65.

83. Avery AJ, Rodgers S, Cantrill JA, et al. A pharmacist-led information technology intervention for medication errors (PINCER): a multicentre, cluster randomised, controlled trial and cost-effectiveness analysis. Lancet. 2012;379(9823):1310-9.

84. Jódar-Sánchez F, Malet-Larrea A, Martín JJ, García-Mochón L, et al. Cost-utility analysis of a medication review with follow-up service for older adults with polypharmacy in community pharmacies in Spain: the conSIGUE program. Pharmacoeconomics. 2015;33(6):599-610.

85. Gallagher J, O'Sullivan D, McCarthy S, et al. Structured pharmacist review of medication in older hospitalised patients: a costeffectiveness analysis. Drugs Aging. 2016;33(4):285-94.

86. Miller R, Darcy CM, Friel ABM, et al. Consultant pharmacist case management of older people in intermediate care: a new innovative model Article. Eur J Pers Cent Healthc. 2016;4(1):46-52.

87. Faria R, Barbieri M, Light K, et al. The economics of medicines optimization: policy developments, remaining challenges and research priorities. Br Med Bull. 2014;111(1):45-61.

88. Loh ZWR, Cheen MHH, Wee HL. Humanistic and economic outcomes of pharmacist-provided medication review in the community-dwelling elderly: a systematic review and meta-analysis. J Clin Pharm Ther. 2016;41(6):621-33.

89. Jokanovic N, Tan EC, Sudhakaran S, et al. Pharmacist-led medication review in community settings: an overview of systematic reviews. Res Social Adm Pharm. 2017;13(4):661-85.

90. Tjia J, Velten SJ, Parsons C, et al. Studies to reduce unnecessary medication use in frail older adults: a systematic review. Drugs Aging. 2013;30(5):285-307. 
91. Riordan DO, Walsh KA, Galvin R, et al. The effect of pharmacist-led interventions in optimising prescribing in older adults in primary care: a systematic review. SAGE Open Med. 2016; $10.1177 \% 2$ F2050312116652568

Publisher's Note Springer Nature remains neutral with regard to jurisdictional claims in published maps and institutional affiliations. 